\title{
A Macromolecular Additive to Turn a Thermoplastic Elastomer into a Self-Healing Material
}

Léo Simonin, ${ }^{a}$ Guillaume Falco, ${ }^{b}$ Sandrine Pensec, ${ }^{a}$ Florent Dalmas, ${ }^{b}$ Jean-Marc Chenal, ${ }^{b}$

François Ganachaud, ${ }^{c}$ Alba Marcellan, ${ }^{d, e}$ Laurent Chazeau ${ }^{b, *}$ and Laurent Bouteiller ${ }^{a, *}$

${ }^{a}$ Sorbonne Université, CNRS, IPCM, Equipe Chimie des Polymères, F-75005 Paris, France

${ }^{\mathrm{b}}$ Univ Lyon, INSA-Lyon, CNRS UMR 5510, MATEIS, F-69621 Villeurbanne, France

`Univ Lyon, INSA Lyon, CNRS UMR 5223, IMP@ INSA-Lyon, F-69621 Villeurbanne, France

${ }^{\mathrm{d}}$ Sciences et Ingénierie de la Matière Molle, ESPCI Paris, PSL University, CNRS, Sorbonne Université, 75005 Paris, France

${ }^{\mathrm{e}}$ Institut Universitaire de France (IUF)

TOC graphic.

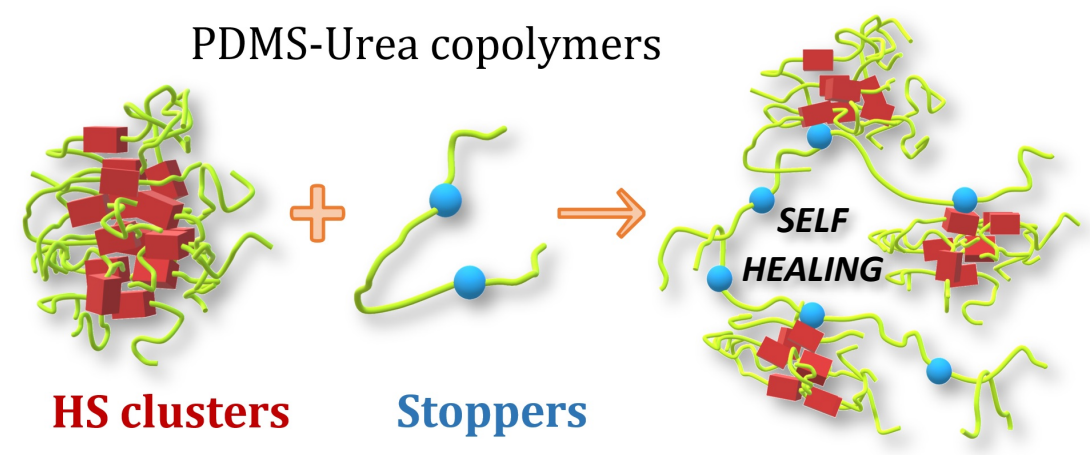


ABSTRACT. Self-healing allows increasing the service life of materials by overcoming some issues caused by mechanical failures. We propose a new concept to bring room temperature selfhealing properties to thermoplastic elastomers. A macromolecular additive whose interacting units can interfere with the hard segments of the thermoplastic elastomer accelerates chain dynamics and brings self-healing properties to the composite material with a limited detrimental effect on mechanical properties. By applying this concept to silicone-based elastomers, we have obtained an autonomously self-healing material with a relatively high elastic modulus for this type of elastomers.

KEYWORDS. silicone, self-healing, chain stopper, hydrogen-bond

\section{INTRODUCTION}

Materials that are able to heal spontaneously have been a long-standing dream of engineers and scientists working in a wide variety of applied fields such as coatings ${ }^{1,2}$, rubbers $^{3}$, composites ${ }^{4}$, asphalt pavements ${ }^{5}$, or cementitious materials ${ }^{6}$. Indeed, self-healing materials can provide a much longer service time and can significantly improve reliability for numerous applications ${ }^{7-9}$. As far as polymer materials are concerned, three main strategies have been explored ${ }^{10}$ : microencapsulation of healing agents ${ }^{11}$, reversible covalent bonds ${ }^{12,13}$ and reversible physical interactions ${ }^{14-16}$. The latter has been naturally adopted when repeated autonomous self-healing is required. Finding a good compromise between healing dynamics and mechanical $\operatorname{strength}^{17}$, necessitates fine-tuning of the structure and dynamics. 
In the case of self-healing elastomers, several concepts have been put forward to obtain both selfhealing and high elastic modulus. For example, it is possible to introduce glassy domains ${ }^{18}$ or particles ${ }^{19}$ in a self-healing elastomer or to combine two networks exhibiting different dynamics ${ }^{20}$. While these approaches have demonstrated some effectiveness, they all share the common feature that the materials were designed from scratch (i.e. they are not based on an existing elastomer), which means a significant cost when industrial production is considered.

A potentially much more practical approach would be to design an additive that could be introduced in a limited amount into an already known elastomer matrix to impart self-healing features to the new composite material. Thermoplastic elastomers (TPEs) that are based on the microphase separation of soft segments and hard segments ${ }^{21}$ are particularly well-suited for such an approach. Processing and recycling of these materials are possible at high temperature thanks to the fast dynamics of the hard segments. In contrast, at room temperature the 'frozen' dynamics of these phase-separated segments maintain the rubbery network topology. The nanostructuration and content of these segments, when controlled, allow a fine adjustment of the mechanical properties of the TPEs (stiffness, strength...), but prevent autonomous self-healing. The addition of a liquid ${ }^{22}$ or a plasticizer ${ }^{23}$ to physically cross-linked elastomers has been reported as a mean to obtain this property, however the resulting faster dynamics of the cross-links leads to a steep decrease in elastic modulus of the material, even at high strain rate.

In order to try to reach a better compromise, we decided to investigate the design of macromolecular additives with two requirements: (1) the presence of suitable interacting groups that could accelerate the dynamics of the hard segments, and (2) a high molar mass that would create new transient cross-links that should help maintain good elastic properties of the final composite material. 


\section{RESULTS AND DISCUSSION}

To test this concept, we have chosen as a matrix a hydrogen-bonded thermoplastic elastomer $(\mathbf{T})$ constituted by the alternation of polydimethylsiloxane (PDMS) soft blocks and bis-urea hard blocks (Figure 1A) ${ }^{24}$. The chosen additive (1) has a structure very similar to the matrix to ensure a good miscibility, except that an ethyl group was introduced on each urea moiety (Figure 1B). Alkylated ureas are good hydrogen bond acceptors but poor hydrogen bond donors; therefore they are expected to interact with the matrix bis-ureas, but disturb the physically elastic network because of the steric hindrance caused by the ethyl groups. Such perturbation is well-known and has been named as a "chain-stopper" effect in the case of low molar mass bis-ureas that form supramolecular polymers in solution ${ }^{25}$, because the alkylated urea groups shorten the chains made of hydrogen bonded ureas (Figure 1C) ${ }^{26,27}$. 
A
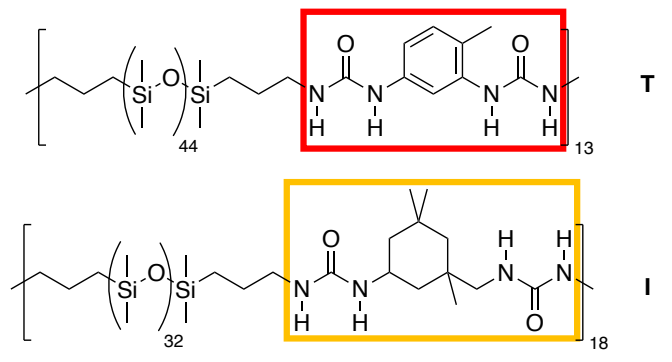

B
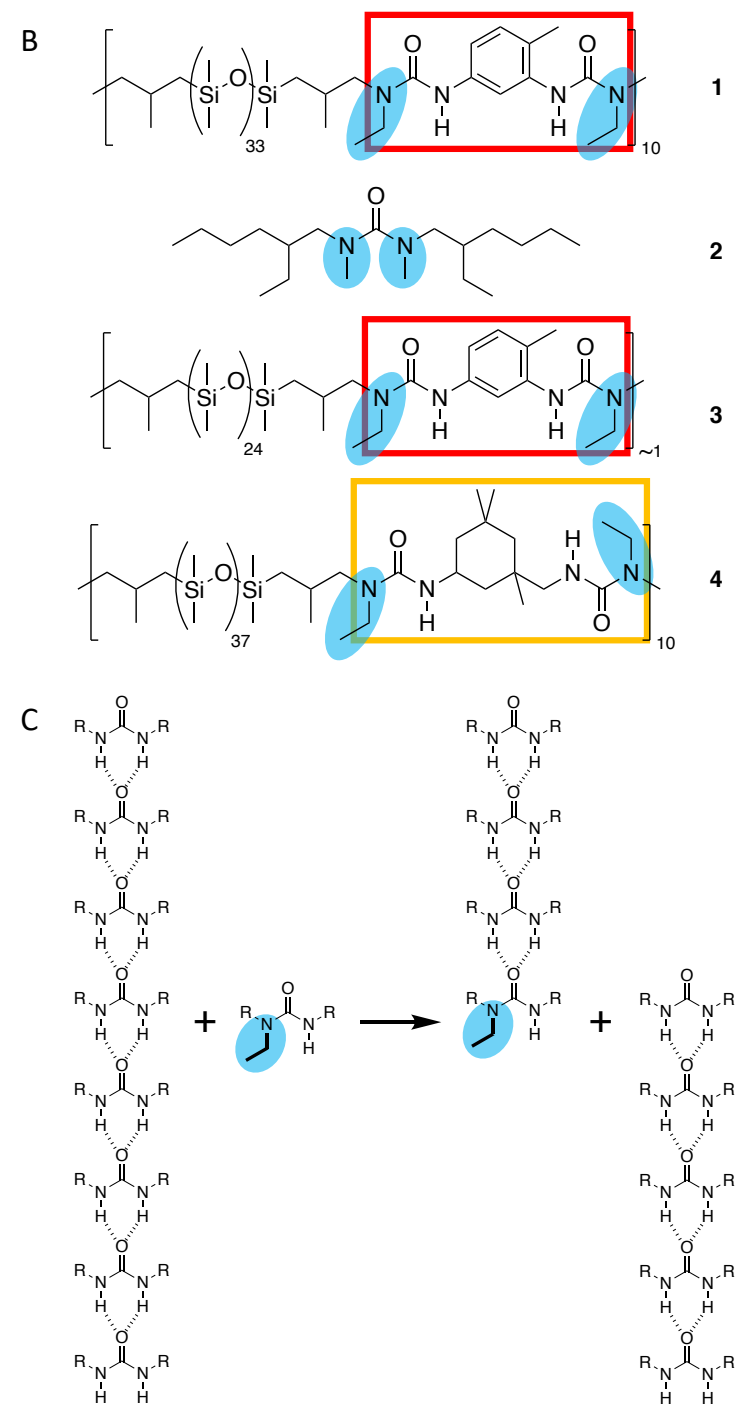

Figure 1. (A) Structure of matrices $\mathbf{T}$ and $\mathbf{I}$ (hydrogen bond stickers are highlighted in red and orange, respectively). (B) Structure of additives (alkylated ureas preventing hydrogen bonding are highlighted in blue). Additives 1, 2 and $\mathbf{3}$ have been tested with matrix $\mathbf{T}$; additive $\mathbf{4}$ concerns matrix I. (C) Illustration of the "chain-stopper" concept. 
Both matrix $\mathbf{T}$ and additive $\mathbf{1}$ were obtained in a single step by polycondensation of toluenediisocyanate (TDI) with telechelic aminopropyl- or ethylaminopropyl-terminated PDMS, respectively ${ }^{28}$. They were dissolved in a common solvent, mixed together and cast as films. The corresponding blend (T1) contains $8 \mathrm{wt} \%$ of additive 1. A low molar mass methylated mono-urea (2) was considered as a reference additive. The corresponding blend (T2) contains $2 \mathrm{wt} \%$ of additive 2, which corresponds to the same molar fraction of added urea groups as in $\mathbf{T 1}$ (10mol\%).

Tensile measurements (Figure 2A and Table 1) show that both additives reduce both stiffness and strength compared to the pure matrix. As expected, the low molar mass additive (blend T2) acts also as a plasticizer, reducing the modulus and involving very large plastic deformation until fracture (strain at break of 2000\%). Interestingly, the addition of the high molar mass additive (blend T1), significantly reduces the stretchability - strain at break is then commensurable with the one of the pure matrix - and implies an increase in the stress at break by 2 -folds compared to blend T2. From the mechanical behaviour at the early stages of deformations, this effect can be related to a significant increase in the yielding stress and the modulus (see insert). Comparatively to the matrix, the macromolecular additive (blend T1) induces a decrease in the tensile modulus of only $20 \%$, i.e. modulus is around $1 \mathrm{MPa}$, whereas the blend containing the low molar mass additive (T2) is characterized by (1) a decrease of 50\% in stiffness, (2) the absence of strain hardening and (3) a large creeping, as visually obvious from the photograph (Figure 2C). 

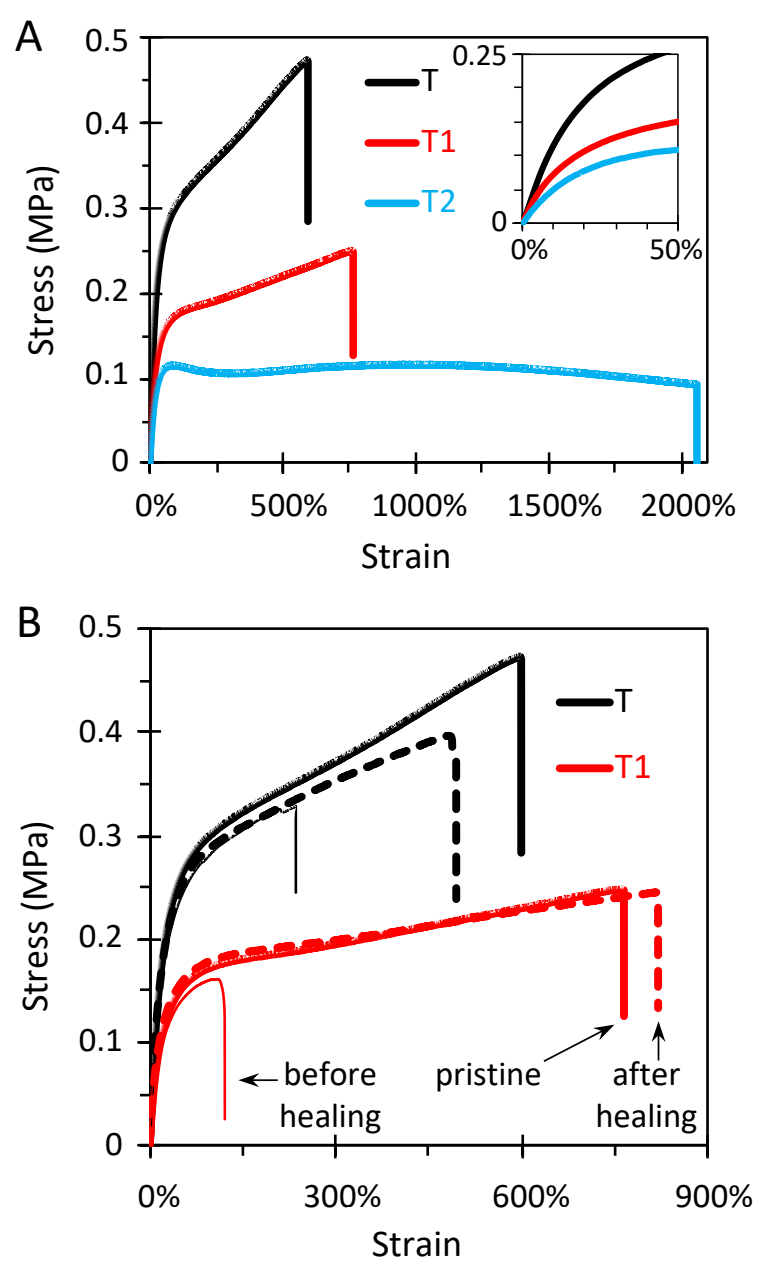

C

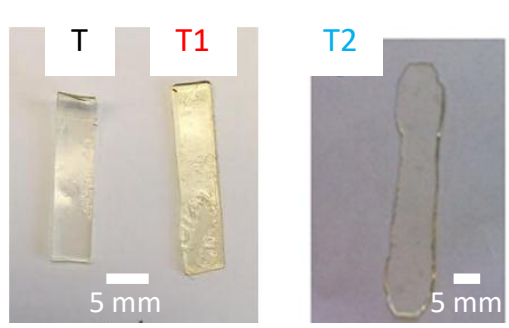

Figure 2. (A,B) Stress-strain curves for the matrix T (black) and blends T1 (red) and T2 (blue). Thick lines are used for uncut (pristine) samples. Dashed (resp. thin) lines are used for samples cut across half their width $24 \mathrm{~h}$ before testing (resp. just before testing). Insert in (A): focus on the low strain region. Measurements and healing were performed at $22^{\circ} \mathrm{C}$. (C) Picture of 
rectangular shaped strips of the matrix $\mathbf{T}$ and the blends $\mathbf{T} 1$ and $\mathbf{T} 2,24 \mathrm{~h}$ (at $22^{\circ} \mathrm{C}$ ) after shaping.

Sample T2 has visibly crept.

Table 1. Mechanical properties of matrices (T, I) and blends (T1, T2, T3, I4).

\begin{tabular}{|c|c|c|c|c|c|}
\hline & & $\begin{array}{l}\text { Tensile modulus } \\
(\mathrm{MPa})\end{array}$ & $\begin{array}{c}\text { Stress at } \\
\text { break }(\mathrm{MPa})\end{array}$ & $\begin{array}{c}\text { Strain at } \\
\text { break (\%) }\end{array}$ & $\begin{array}{l}\text { Self-healing } \\
\text { efficiency }(\%)^{\mathrm{c}}\end{array}$ \\
\hline \multirow{3}{*}{$\mathbf{T}$} & pristine & 1.20 & $0.47 \pm 0.05$ & $600 \pm 60$ & \multirow{3}{*}{50} \\
\hline & healed $^{\mathrm{a}}$ & & $0.40 \pm 0.04$ & $490 \pm 50$ & \\
\hline & freshly cut ${ }^{\mathrm{b}}$ & & $0.33 \pm 0.03$ & $230 \pm 30$ & \\
\hline \multirow{3}{*}{ T1 } & pristine & 0.96 & $0.27 \pm 0.03$ & $770 \pm 80$ & \multirow{3}{*}{90} \\
\hline & healed $^{\mathrm{a}}$ & & $0.26 \pm 0.03$ & $820 \pm 90$ & \\
\hline & freshly cut ${ }^{\mathrm{b}}$ & & $0.17 \pm 0.02$ & $110 \pm 20$ & \\
\hline T2 & pristine & 0.59 & $0.12 \pm 0.01$ & $2050 \pm 200$ & \\
\hline T3 & pristine & 0.65 & $0.26 \pm 0.03$ & $640 \pm 70$ & \\
\hline I & pristine & 3.6 & $2.9 \pm 0.3$ & $480 \pm 50$ & \\
\hline I4 & pristine & 1.9 & $1.9 \pm 0.2$ & $470 \pm 50$ & \\
\hline
\end{tabular}

${ }^{a}$ Samples were cut across half their width and tested after resting $24 \mathrm{~h}$ at $22^{\circ} \mathrm{C}$.

${ }^{\mathrm{b}}$ Samples were cut across half their width and immediately tested.

${ }^{\mathrm{c}}$ Measured as the stress at break recovery: $\left(\sigma_{\text {healed }}-\sigma_{\text {freshly cut }}\right) /\left(\sigma_{\text {pristine }}-\sigma_{\text {freshly cut }}\right)$ 

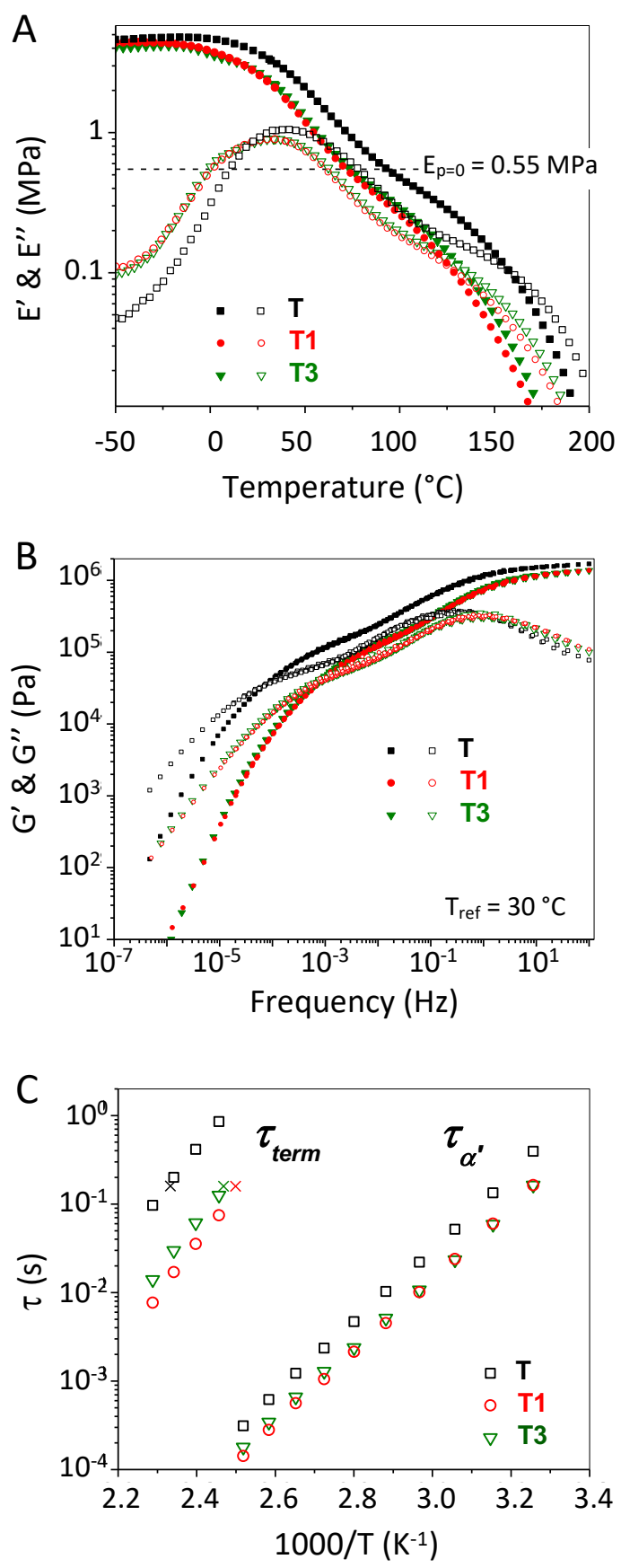

Figure 3. Dynamics of the matrix $\mathbf{T}$ (black squares) and blends $\mathbf{T 1}$ (red circles) and $\mathbf{T 3}$ (green triangles) obtained by combining DMA and rheology measurements: (A) Storage moduli (filled symbols) and loss moduli (open symbols) versus temperature (heating rate: $\left.2^{\circ} \mathrm{C} \cdot \mathrm{min}^{-1}\right)$; (B) Master curves at $30^{\circ} \mathrm{C}$ (same symbols); (C) Characteristic relaxation times for the $\alpha$ ' process 
$\left(\tau_{\alpha^{\prime}}\right)$ and the terminal relaxation process $\left(\tau_{\text {term }}\right)$ resulting from the building of the master curves. The $\tau_{\text {term }}$, resulting from the isochrones $G^{\prime} / G^{\prime \prime}$ crossover $(A)$ are added as cross symbols.

To probe self-healing, samples were cut across half their width, the created surfaces were then put back into contact and tensile measurements were done immediately after, or $24 \mathrm{~h}$ after cutting (Figure 2B). Healing efficiency was estimated from the stress at break recovery versus the freshly cut samples, using the pristine sample to normalize the data (see Table 1 for the precise definition). The pure matrix exhibits limited self-healing properties with an efficiency of $50 \%$ after $24 \mathrm{~h}$ at $22^{\circ} \mathrm{C}$, which shows that hydrogen bond dynamics in the pure matrix is slow but not completely frozen $^{22}$. This result is significantly improved in the case of the blend $\mathbf{T 1}$ for which the healing efficiency reaches $90 \%$ at the same temperature ${ }^{29}$. Healing kinetics is thus faster for the blend T1 than for the matrix $\mathbf{T}$. Therefore, the high molar mass additive $\mathbf{1}$ improves self-healing kinetics, but unlike the low molar mass additive $\mathbf{2}$, it does not act as a plasticizer. The tensile behaviour is qualitatively similar and only a moderate decrease of tensile modulus is measured compared to the pure matrix.

In the following, we further investigate these materials by looking at various parameters influencing self-healing properties, namely chain dynamics, and phase separation (incompatibility and structure). The first phenomenon studied was the chain dynamics. Figure 3A shows isochronal curves from combined DMA and rheology measurements in the -50 to $200{ }^{\circ} \mathrm{C}$ domain. For $-50<$ $\mathrm{T}<-20{ }^{\circ} \mathrm{C}$, the matrix $\mathbf{T}$ and the blend $\mathbf{T} 1$ show a plateau with a similar storage modulus $\left(E^{\prime} \sim 4.5\right.$ $-5 \mathrm{MPa}$ ), in line with tensile experiments ${ }^{30}$. The modulus value is the consequence of (i) the formation of physical crosslinks formed by the associated stickers effectively permanent over an 
association time that is larger than the observation time, and (ii) the phase separation of the stickers which can lead to the formation of a reinforcing structure (filaments as shown later) ${ }^{24}$. For $\mathrm{T}>-20$ ${ }^{\circ} \mathrm{C}$, the matrix and the blend $\mathbf{T} \mathbf{1}$ show two consecutive relaxation processes as noticed by two drops in G', shifted to lower temperatures for the blend. The first relaxation $\alpha^{\prime}$ is assigned to the dynamics of the hard domains, i.e. a cascade of lifetimes of the sticker association ${ }^{24}$. By increasing the temperature, this lifetime is decreased, as well as the effective number of mechanically effective stickers within the observation timescale. This decrease is followed by a pseudo-plateau, and finally by the flowing of polymer chains. Introduction of the additive in the blend decreases the temperature of the $\alpha^{\prime}$ relaxation, suggesting a shorter sticker association lifetime. Similar observations arise from the temperature of the terminal relaxation ( $E^{\prime} / E^{\prime \prime}$ crossover temperature), which is decreased by $37{ }^{\circ} \mathrm{C}$ (at $1 \mathrm{~Hz}$ ) for $\mathbf{T 1}$ compared to the matrix $\mathbf{T}$. The evolution of the materials relaxation times with temperature were more deeply characterized by frequency sweeps at different temperatures (Figure S6). Rheological master curves were built at a reference temperature of $30^{\circ} \mathrm{C}$ i.e., around the temperature at the $\mathrm{E}$ ' maximum on the isochronal curve (Figure 3B). As shown in this figure, time-temperature superposition principle works remarkably well, suggesting that there is no sharp change in the relaxation processes in the whole temperature range. Regarding the terminal relaxation, as expected, the slopes tend toward 2 and 1 for $\mathrm{G}^{\prime}$ and $\mathrm{G}^{\prime \prime}$, respectively. From the master curves, it is possible to estimate that the $\mathrm{G}^{\prime} / \mathrm{G}^{\prime \prime}$ crossover frequencies are $7.310^{-5}$ and $8.410^{-4} \mathrm{~Hz}$ for $\mathbf{T}$ and $\mathbf{T 1}$, respectively (at a reference temperature of $30^{\circ} \mathrm{C}$ ). This estimation shows the impact of the additive on the terminal relaxation of the material: the blend $\mathbf{T 1}$ exhibits a crossover frequency one order of magnitude higher than the corresponding matrix, reflecting the enhanced dynamics introduced by the additive. This result is in perfect agreement with the improved self-healing of the blend demonstrated at a similar temperature 
$\left(22^{\circ} \mathrm{C}\right)$. To go a step further, the relaxation times deduced by building the master curves are reported in Figure 3C. The relaxation times $\tau_{\alpha}$, are estimated from the frequency at the maximum of the $G^{\prime \prime}$ peak and the terminal relaxation times $\tau_{\text {term }}$, at the time of $G^{\prime} / G^{\prime \prime}$ crossover. From the Arrhenius fitting of these characteristic times, activation energies $\left(E_{a}\right)$ can be estimated. For the $\alpha$ ' process, $\mathrm{E}_{\mathrm{a}}$ is found very similar for the matrix and the blend $\left(\mathrm{ca} .78 \mathrm{~kJ}^{\mathrm{mol}}{ }^{-1}\right)$. This activation energy is consistent with the activation energy of bidentate hydrogen bonding $\left(59 \mathrm{~kJ}^{\mathrm{mol}}{ }^{-1}\right)^{31}$. An activation energy can also be deduced for $\tau_{\text {term }}\left(108 \mathrm{~kJ} \mathrm{~mol}^{-1}\right)$ which is slightly larger than the one calculated for $\tau_{\alpha^{\prime}}$, as seen in Figure 3C. Once again, the values are similar for the matrix $\mathbf{T}$ and the blend T1. A more interesting result is that introducing the additive has more impact on the terminal relaxation times. Indeed, whereas $\tau_{\alpha^{\prime}}$ was decreased by a factor 2 for the blend, $\tau_{\text {term }}$ is decreased by a factor 10 , indicating much faster chain dynamics. $\tau_{\text {term }}$ is often closely connected to healing time, and the extrapolation of $\tau_{\text {term }}$ at $25^{\circ} \mathrm{C}\left(1 / \mathrm{T}\right.$ then equal $\left.3.35 \times 10^{-3} \mathrm{~K}^{-1}\right)$ provides an estimation of the required time for the material to heal. Consequently, the lower value of $\tau_{\text {term }}$ for the blends may explain the faster healing kinetics observed for $\mathbf{T} 1$ compared to the matrix $\mathbf{T}$. To sum up, the relaxation mechanisms of the blend are accelerated by the addition of the supramolecular additive while keeping mechanical properties at conventional strain rates very similar to the neat matrix (Figure 2A).

Next, the impact of the additive on the micro-phase separation was analysed to check if the observed change in dynamics could be ascribed to a change in microstructure. Figures 4A,B display ambient tapping-mode AFM phase images recorded from the films cross-section, while Figure 4C shows the corrected scattered intensity I(q) obtained from USAXS and SAXS experiments. Concerning the matrix $\mathbf{T}$, the AFM data shows microphase separation into filaments of random orientation and with an average inter-filament distance on the order of 15-20 nm. The 
X-ray scattered intensity of the matrix shows at low $\mathrm{q}\left(\mathrm{q}<0.10 \mathrm{~nm}^{-1}\right)$, a $\mathrm{q}^{-3.3}$ regime, which may be due to a 3D heterogeneity on a large scale (i.e. more or less organized bundles or domains). The explored q domain does not cover small enough q values to extract a characteristic dimension for these scattering objects. Nevertheless, it is possible to evaluate a correlation distance from X-ray measurements: subtraction of the fitted curve at low q reveals a weak peak centred at $\mathrm{q} \sim 0.3 \mathrm{~nm}^{-}$ 1. This value corresponds to ca. $22 \mathrm{~nm}$, which is close to the distance between filaments evaluated from AFM. This therefore confirms our interpretation. Another peak is evidenced at larger q, with a maximum at $\mathrm{q} \sim 1.45 \mathrm{~nm}^{-1}$. This distance $(4.3 \mathrm{~nm})$ is close to the end-to-end distance of the PDMS soft blocks calculated with the freely rotating chain model $(3.3 \mathrm{~nm})^{32}$. We therefore assign it to the distance between the hard blocks of the segmented PDMS-urea copolymer ${ }^{24}$. Since the distance between filaments $(22 \mathrm{~nm})$ is longer than the end-to-end distance of the soft blocks $(4.3$ $\mathrm{nm})$, it means that some stickers are trapped into the soft phase probably in the form of small clusters with dimensions way lower than those of the filaments observed in AFM.

Blend T1, also exhibits a filament-like microstructure (Figure 4B) but with a more homogeneous spatial distribution. The less defined nanostructuration in filaments is confirmed by the SAXS/USAXS pattern (Figure 4C) where no peak can be extracted at $\mathrm{q} \sim 0.3 \mathrm{~nm}^{-1}$. Other than that, the blend exhibits the same features as the matrix chains, i.e. a peak at $\mathrm{q} \sim 1.45 \mathrm{~nm}^{-1}$ and a $\mathrm{q}^{-3.3}$ dependence at low q value however shifted to smaller q. Therefore, strong microphase separation occurs also for the blend, and the improved self-healing kinetics brought by the additive, which is related to faster chain dynamics, comes from a less constrained microstructure. 

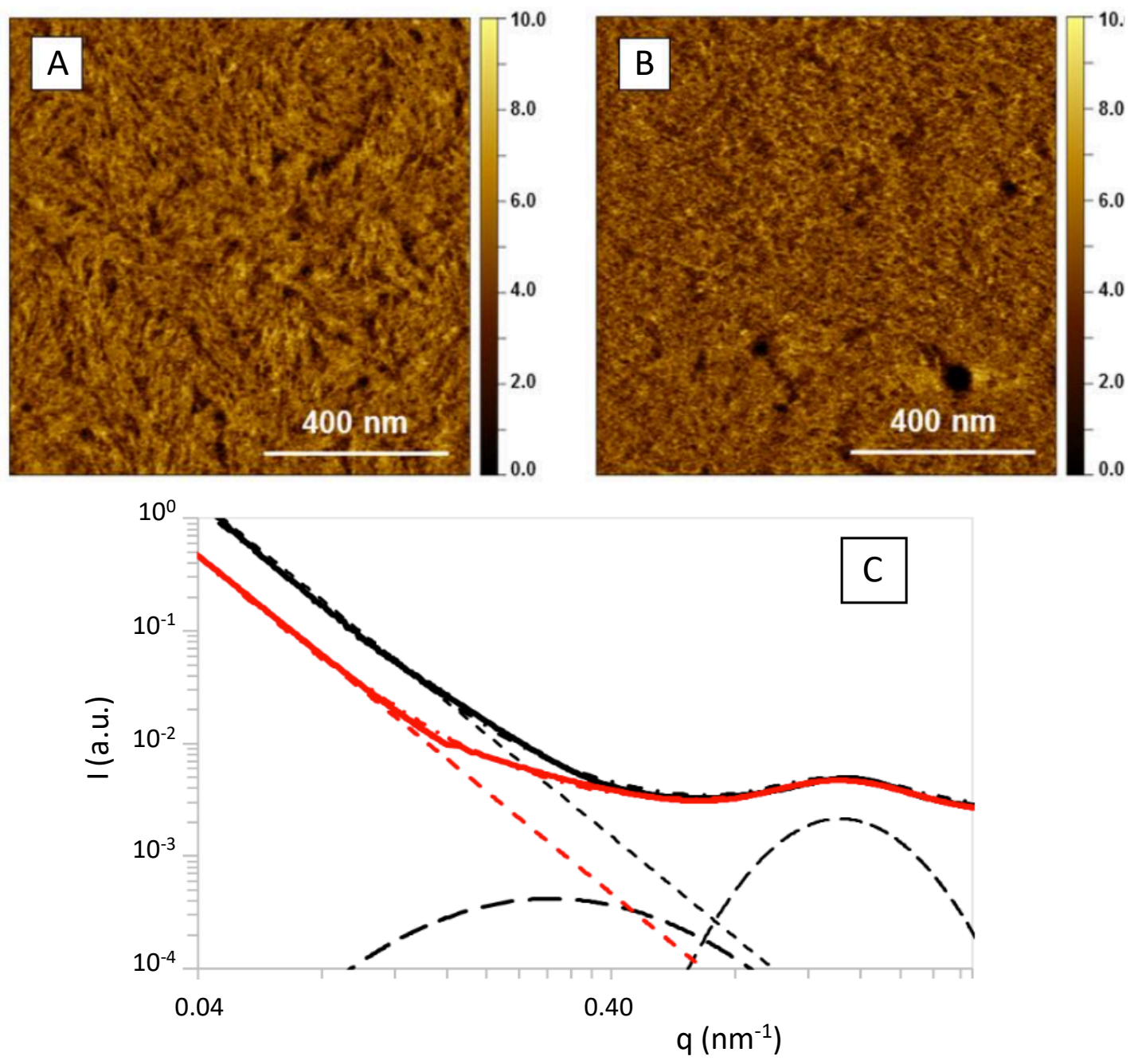

Figure 4. Tapping-mode AFM phase images at room temperature of the matrix $\mathbf{T}(\mathrm{A})$ and the blend T1 (B) (films cross-section). (C) Scattered intensity (azimuthally integrated signal from the isotropic 2D scattering pattern) of the matrix $\mathbf{T}$ (black) and the blend $\mathbf{T} 1$ (red). Fits (dashdotted lines): the matrix $\mathbf{T}$ is fitted thanks to the summation of a $\mathrm{q}^{-3.3}$ exponential decay and two peaks (the low q peak being of very weak intensity); only one peak is necessary for the blend T1 with the $\mathrm{q}^{-3.3}$ decay, to perfectly fit the $\mathrm{I}(\mathrm{q})$ curve (it is the same as the high q peak used for the fit of the $\mathbf{T}$ curve). 
The striking difference between additives $\mathbf{1}$ and $\mathbf{2}$ (see Figure 2C) prompted us to study the impact of the additive molar mass on the balance between stiffness and self-healing of the blends. Additive 3 was synthesized from the same reactants as additive 1, but with an excess of soft block precursor in order to obtain much shorter chains. Additive $\mathbf{3}$ contains an average number of stickers per chain 10 times lower than 1 (Table S1) ${ }^{33}$. The corresponding blend (T3) contains 8 wt\% of additive $\mathbf{3}$, i.e. the same weight fraction of additive as in T1. Stress-strain curves (Figure 5) obtained at a strain rate of $0.06 \mathrm{~s}^{-1}$ show some differences between the blends $\mathbf{T 3}$ and $\mathbf{T 1}$. As reported in Table 1, the stresses at break are almost the same for both blends, but $\mathbf{T 1}$ exhibits a higher tensile modulus compared to $\mathbf{T 3}$. Such result is not surprising considering that $\mathbf{T 3}$ contains smaller and more mobile chains. In addition, this smaller additive is able to connect fewer supramolecular clusters than the longer one, which may also explain the decrease of tensile modulus observed from $\mathbf{T} 1$ to $\mathbf{T} 3^{34}$. In contrast, the temperature sweep rheological measurements for both blends (Figure 3A) are very similar, except at low temperature (below $25^{\circ} \mathrm{C}$ ), where the slightly higher elastic modulus of $\mathbf{T 1}$ confirms the tensile modulus results. Frequency sweep rheology (Figure 3B) also shows that the additive molar mass has a moderate impact on chain dynamics, with similar crossover $\mathrm{G}^{\prime} / \mathrm{G}^{\prime \prime}$ frequencies for $\mathbf{T 3}\left(7.610^{-4} \mathrm{~Hz}\right)$ compared to $\mathbf{T 1}\left(8.410^{-4} \mathrm{~Hz}\right)$. Apparently using an additive with a higher molar mass (T1) brings better results in the balance between stiffness and chain dynamics. 


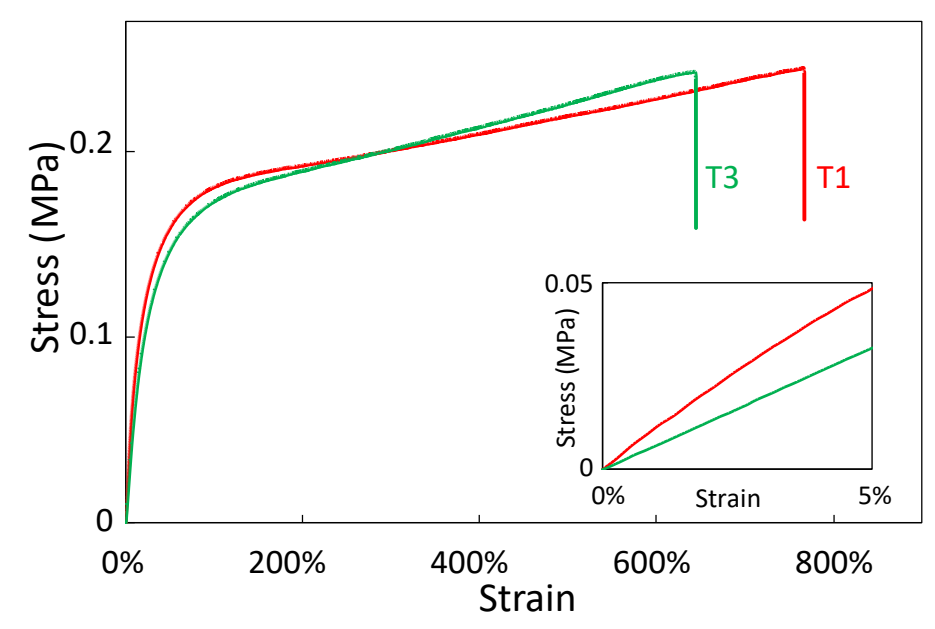

Figure 5. Stress-strain curves for blends $\mathbf{T 1}$ (red) and $\mathbf{T 3}$ (green) $\left(22^{\circ} \mathrm{C}\right)$. The insert highlights the initial slopes.

Figure 6 provides a molecular representation consistent with our results. As shown by the SAXS and AFM data (Figure 4), the presence of the additive reduces the size of the hydrogen bonded clusters, which in turn accelerates the relaxation mechanisms probed by mechanical spectroscopy (Figure 3). Remarkably, the acceleration occurs independently from the molar mass of the additive, while the stiffness of the blend is improved for the additive with the highest molar mass (Figure $5)$.

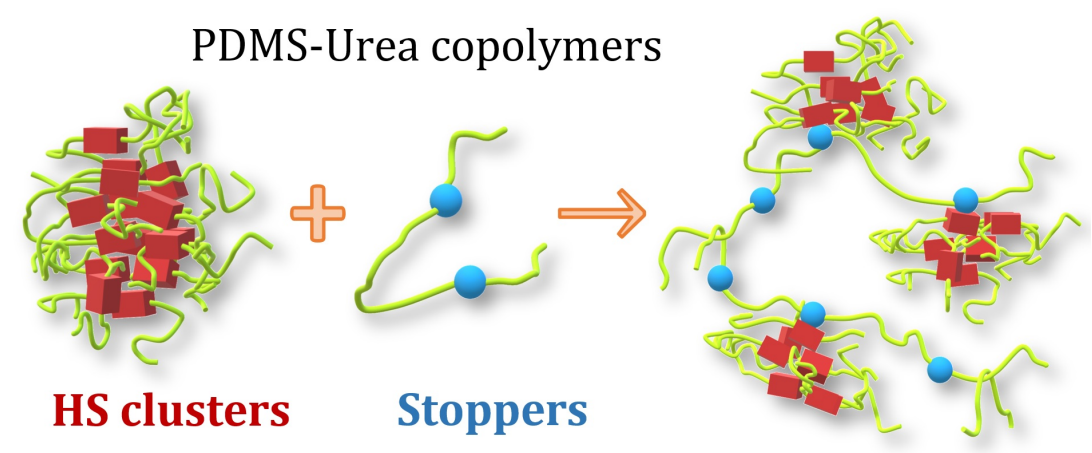

Figure 6. Cartoon representation of the effect of the chain stopper additive. The alkylated urea stickers (blue spheres) of the additive interfere with the hard domains (red blocks) of the matrix 
but if the additives have a high molar mass, they can still provide connections between hydrogen bonded clusters.
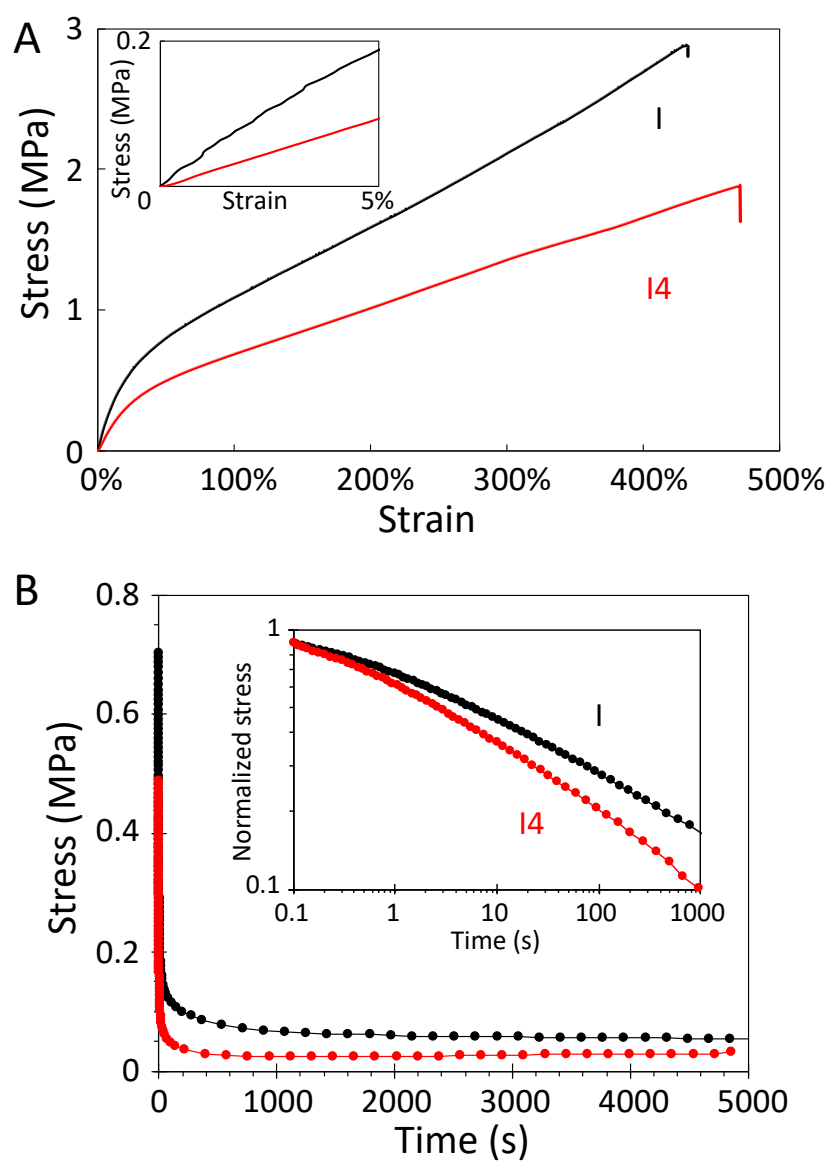

C
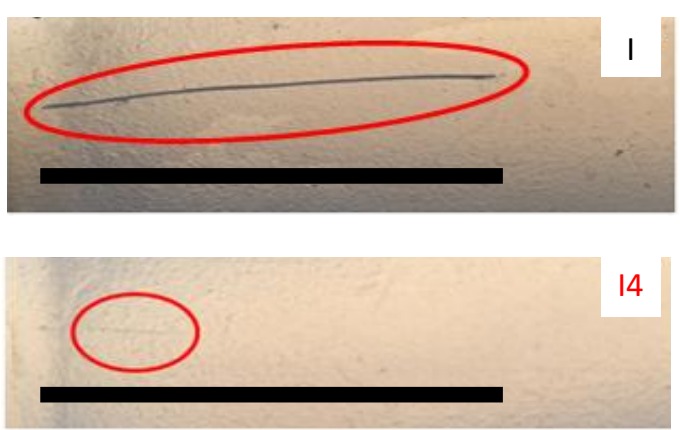

Figure 7. Mechanical properties for matrix I (black) and blend I4 (red). (A) Stress-strain curves $\left(22^{\circ} \mathrm{C}\right)$. The insert shows the initial slopes. (B) Stress relaxation curves at $22^{\circ} \mathrm{C}$. The insert shows the $\log$ - $\log$ stress relaxation curves. (C) Self-healing results after 6 days at room temperature. 
The black line $(2.5 \mathrm{~cm})$ represents the length of the original cut (that was made across the whole thickness of the film).

In order to prove the generality of the concept, an analogous but stiffer matrix was selected (I, Figure 1). This isophoronediisocyanate (IPDI) based PDMS-urea segmented copolymer, is a commercially available material (GENIOMER $\left.{ }^{\circledR} 80\right)$. The structure of the additive (4) was adapted to the matrix (Figure 1) to favour miscibility, and mechanical and self-healing tests were performed on the matrix I and the blend $\mathbf{I} 4$ that contains $10 \mathrm{wt} \%$ of additive 4. Strain-strain curves, reported in Figure 7A, lead to the same observations made for TDI-based materials: the additive moderately decreases the tensile modulus and the strain at break (Table 1). Meanwhile, stress relaxation behaviour (Figure 7B) seems to exhibit faster processes at long timescale ( $\mathrm{t}>100 \mathrm{~s})$ for the blend than for the matrix ${ }^{35}$. With lower relaxation time for the blend, a faster self-healing may be expected.

Self-healing was tested at room temperature: matrix and blend samples were cut with a razor blade and kept at room temperature. After 6 days it is clear (Figure 7C) that the matrix I does not show any healing, whereas the cut has almost entirely disappeared for the blend I4. This observation confirms that the additive brings self-healing properties with only a limited decrease of the rigidity of the initial thermoplastic elastomer.

\section{CONCLUSION}

We have demonstrated a new concept (Figure 6) to bring self-healing properties to a thermoplastic elastomer. Adding a polymer with interacting units that can interfere with the thermoplastic elastomer hard segments allows accelerating chain dynamics while showing limited detrimental 
effect on the rigidity. We have applied this concept to silicone based elastomers and obtained an autonomously self-healing material with an elastic modulus of $1.9 \mathrm{MPa}$, which is a remarkable feature for this type of elastomers deprived of inorganic fillers ${ }^{363738}$. Future works will focus on the use of such additives on the formulation of other segmented copolymers using supramolecular interactions.

\section{EXPERIMENTAL SECTION}

Matrix synthesis: The material sources and experimental procedures are provided in supporting information. In short, matrix $\mathbf{T}$ was synthesized by addition of $\alpha, \omega$-bis $(\gamma$ aminopropyl)poly(dimethylsiloxane) $\left(\mathrm{M}_{\mathrm{n}}=3240 \mathrm{~g} \cdot \mathrm{mol}^{-1}\right)$ to TDI in a THF solution, with a yield of $82 \%$.

Additives synthesis: The additives were synthesized by a similar procedure from the relevant diisocyanate (TDI or IPDI) and N-ethylaminoisobutyl terminated polydimethylsiloxane $\left(\mathrm{M}_{\mathrm{n}}=\right.$ $\left.2430 \mathrm{~g} \cdot \mathrm{mol}^{-1}\right)$.

Additive/matrix formulation and film preparation: Blends were prepared by solvent casting. A calculated amount of matrix and additive were dissolved independently in tetrahydrofuran $(10 \mathrm{~mL}$ of THF per gram of polymer). After stirring for 5 hours, the two solutions were combined and stirred for one night. Then the solution was poured into a partially closed polypropylene mold to allow slow solvent evaporation. After 1 day, the mold was open and the film was left 1 more day at room temperature. Then the mold was placed in a vacuum oven $\left(10^{-3} \mathrm{mbar}, 60^{\circ} \mathrm{C}\right)$ for 1 day. After the thermal treatment, the films were removed from the mold and stored at room temperature. The film thickness was measured to in between 0.5 and $1 \mathrm{~mm}$. 
Tensile testing: Tensile tests were performed on a standard tensile Instron machine, model 5565. The device used a $100 \mathrm{~N}$ load cell (with a relative uncertainty of $0.1 \%$ in the range from 1 to 100 N) and a video extensometer which follows the local displacement up to $120 \mathrm{~mm}$ (with a relative uncertainty of $0.11 \%$ at full scale). Monotonic tensile tests were carried out on strip shaped samples. They were cut from the films and had a reduced section of $5 \mathrm{~mm}$-width $\mathrm{x} 0.5$ to $1 \mathrm{~mm}$ thickness. The gauge length (initial distance between jaws) was taken constant $\left(\mathrm{L}_{0} \sim 20 \mathrm{~mm}\right.$ ) for all the tests. Tests were carried out at a velocity of $1.2 \mathrm{~mm} \cdot \mathrm{s}^{-1}$ (corresponding to a nominal strain rate of $0.06 \mathrm{~s}^{-1}$ ) until failure. All samples were marked with two dots within the reduced section, for their recognition by the video extensometer. During the test, the force $(\mathrm{F})$ and the displacement $(\Delta \mathrm{L})$ were recorded and the nominal stress $\left(\sigma=\mathrm{F} / \mathrm{S}_{0}\right)$ and the nominal strain $\varepsilon\left(\varepsilon=\Delta \mathrm{L} / \mathrm{L}_{0}\right)$ were calculated. Regarding stress relaxation experiments, the samples were stretched up to $20 \%$ strain at a velocity of $10 \mathrm{~mm} \cdot \mathrm{s}^{-1}$ (corresponding to a nominal strain rate of $0.5 \mathrm{~s}^{-1}$ ), then the strain was fixed over time and the resulting stress was recorded. Initial time was set when the stress reaches its maximal value.

Mechanical spectroscopy: Mechanical spectroscopy measurements were conducted to determine moduli of the matrix and the blends from -50 to $200^{\circ} \mathrm{C}$. The Dynamic Mechanical Analysis (DMA) was carried out from -50 to $25^{\circ} \mathrm{C}$ while rheological analysis was used for higher temperatures. The DMA experiments were performed in tensile mode on a TA Instruments DMA Q800 under $\mathrm{N}_{2}$ atmosphere with a $2{ }^{\circ} \mathrm{C} \cdot \mathrm{min}^{-1}$ heating rate and $0.1 \%$ strain amplitude. The sample dimensions were 6.00 (length), $3.00 \mathrm{~mm}$ (width) and $0.70 \mathrm{~mm}$ (thickness) $( \pm 0.01 \mathrm{~mm})$. Rheological measurements were done on an Anton Paar MCR 702 TwinDrive at $2{ }^{\circ} \mathrm{C} \cdot \mathrm{min}^{-1}$ under $\mathrm{N}_{2}$ atmosphere. A $25 \mathrm{~mm}$ parallel plate with $0.5 \mathrm{~mm}$ gap, a constant normal force of $0.1 \mathrm{~N}$ and $1 \%$ oscillatory shear deformation were applied. Each sample was placed on the lower plate at $160{ }^{\circ} \mathrm{C}$ before reducing 
the gap by bringing down the upper plate. The $G^{\prime}$ and $G$ ', moduli thus collected were multiplied by a factor of 3 in order to be superposed to E' and E', values obtained by DMA. No other shift of the moduli values was used to correlate DMA and rheology data. The rheological instrument was also used to build master curves for the samples. Samples were subjected to $1 \%$ oscillatory shear deformation over a wide temperature range (from 30 to $160^{\circ} \mathrm{C}$ ). The sample was annealed at $160{ }^{\circ} \mathrm{C}$ and frequency sweeps were isothermally carried every $10^{\circ} \mathrm{C}$ down to $30^{\circ} \mathrm{C}$. A frequency sweep ranging from 100 to $0.1 \mathrm{~Hz}$ was applied (100 to $0.01 \mathrm{~Hz}$ for $160^{\circ} \mathrm{C}$ ). Five points were recorded by decade distributed according to a logarithmic scale. Resulting curves were only shifted along the frequency axis using time-temperature superposition (TTS) to build master curves. The same shift factors were used for matrix $\mathbf{T}$ and blends $\mathbf{T 1}$ and $\mathbf{T 3}$.

Self-healing experiments: Two procedures were used to estimate self-healing efficiency:

- Strip shaped samples were cut, but only half-way across their width, so that the cut surfaces were automatically placed in an optimum position. We found this procedure improved reproducibility of the self-healing test, compared to fully cut samples for which perfect contact is not always obtained. These half-cut samples were left for $24 \mathrm{~h}$ at room temperature with the cut surfaces in close contact. Then tensile measurements were done, and healing efficiency was measured as the stress at break recovery. Of course, it is necessary to take into account the fact that the tensile strength of the freshly cut sample ( $\left.\sigma_{\text {freshly cut }}\right)$ is not negligible. Therefore, the efficiency of healing was calculated as: $\left(\sigma_{\text {healed }}-\sigma_{\text {freshly cut }}\right) /\left(\sigma_{\text {pristine }}-\sigma_{\text {freshly cut }}\right)$.

- Samples were cut directly into the mold used for the shaping. A slit $(2.5 \mathrm{~cm})$ was made with a razor blade, and then a plate of the same dimension as the sample (weight:10g) was placed on the 
top of the cut sample, to allow optimal contact between cut interfaces. Healing efficiency was calculated from the decrease of the slit length.

Atomic force microscopy (AFM): AFM was conducted on a Dimension 3100 AFM device connected to a Nanoscope V scanning probe controller (VEECO Instruments, Plainview, NY). All images were obtained at ambient temperature in tapping mode using a pointprobe-plus ${ }^{\circledR}$ silicon (PPP-NCH-50) from Nanosensors with a high resonance frequency of about $300 \mathrm{kHz}$. A lateral resolution of 3-5 $\mathrm{nm}$ can be achieved by this configuration with a vertical resolution of $0.5 \mathrm{~nm}$. Surfaces of bulk samples were prepared by cryo-ultramicrotomy on a Leica UC7 microtome at $150^{\circ} \mathrm{C}$ using a diamond knife from Diatome. All AFM images thus show the bulk microstructure. Very flat surfaces can be obtained with this technic and only phase images (highlighting a hard/soft contrast) are shown.

Static small angle X-ray scattering (SAXS) and ultra-small angle X-ray scattering (USAXS): SAXS and USAXS experiments were performed on the ID2 beamline of the European Synchrotron Radiation Facility (ESRF), Grenoble (France). For SAXS measurements, the sample-detector distance was 1 meter and the exposure time was set at 0.5 second while for the USAXS investigations, the distance between sample and detector was 10 meters and the exposure time was set at 0.05 second. A small shift has been applied on USAXS y-abscissa data to obtain an optimal superposition with SAXS data in both common q domains. The SAXS/USAXS two-dimensional (2D) patterns of samples have not shown any preferential orientation of the samples.

\section{ASSOCIATED CONTENT}




\section{Supporting Information.}

The following file is available free of charge. Synthesis and additional rheology data (PDF file). AUTHOR INFORMATION

\section{Corresponding Author}

* L.B. e-mail: laurent.bouteiller@ sorbonne-universite.fr

* L.C. e-mail: laurent.chazeau@insa-lyon.fr

\section{Author Contributions}

The manuscript was written through contributions of all authors. All authors have given approval to the final version of the manuscript.

\section{ACKNOWLEDGMENT}

This work was supported by the French Agence Nationale de la Recherche (project ANR-15CE08-0022 Arcade). Wacker Chemie AG is acknowledged for the gifts of the aminopropylpolysiloxane reagent and Geniomer ${ }^{\circledR} 80$. The authors are indebted to the "Microstructure Technological Center" (CT $\mu$ ) of University of Lyon for the access to the transmission electron microscope and the ultramicrotome. We would like also to thank CLYM (www.clym.fr) for the access to their AFM device. We are also indebted to Dr. F Rousset for his precious support for the rheology experiments, and to Dr. G. Stocklet, who generously provided us SAXS beam time.

\section{REFERENCES}

(1) Cho, S. H.; White, S. R.; Braun, P. V. Self-Healing Polymer Coatings. Adv. Mater. 2009, 21 (6), 645-649. https://doi.org/10.1002/adma.200802008.

(2) García, S. J.; Fischer, H. R.; van der Zwaag, S. A Critical Appraisal of the Potential of Self 
Healing Polymeric Coatings. Prog. Org. Coat. 2011, 72 (3), 211-221. https://doi.org/10.1016/j.porgcoat.2011.06.016.

(3) Cordier, P.; Tournilhac, F.; Soulie-Ziakovic, C.; Leibler, Ludwik. Self-Healing and Thermoreversible Rubber from Supramolecular Assembly. Nature 2008, 451, 977-980. https://doi.org/10.1038/nature06669.

(4) Hillewaere, X. K. D.; Du Prez, F. E. Fifteen Chemistries for Autonomous External SelfHealing Polymers and Composites. Prog. Polym. Sci. 2015, 49-50, 121-153. https://doi.org/10.1016/j.progpolymsci.2015.04.004.

(5) Tabakovic, A.; Schlangen, Erik. Self-Healing Technology for Asphalt Pavements. $A d v$. Polym. Sci. 2016, 273, 285-306. https://doi.org/10.1007/12_2015_335.

(6) Li, W.; Dong, B.; Yang, Z.; Xu, J.; Chen, Q.; Li, H.; Xing, F.; Jiang, Z. Recent Advances in Intrinsic Self-Healing Cementitious Materials. Adv. Mater. 2018, 30, 1705679. https://doi.org/10.1002/adma.201705679.

(7) Chen, D.; Wang, D.; Yang, Y.; Huang, Q.; Zhu, S.; Zheng, Z. Self-Healing Materials for Next-Generation Energy Harvesting and Storage Devices. Adv. Energy Mater. 2017, 7, 1700890. https://doi.org/10.1002/aenm.201700890.

(8) Levchenko, I.; Bazaka, K.; Belmonte, T.; Keidar, M.; Xu, S. Advanced Materials for NextGeneration Spacecraft. Adv. Mater. 2018, 30, 1802201. https://doi.org/10.1002/adma.201802201.

(9) Tan, Y. J.; Wu, J.; Li, H.; Tee, B. C. K. Self-Healing Electronic Materials for a Smart and Sustainable Future. ACS Appl. Mater. Interfaces 2018, 10, 15331-15345. https://doi.org/10.1021/acsami.7b19511.

(10) An, S. Y.; Arunbabu, D.; Noh, S. M.; Song, Y. K.; Oh, J. Kwon. Recent Strategies to Develop Self-Healable Crosslinked Polymeric Networks. Chem. Commun. 2015, 51, 1305813070. https://doi.org/10.1039/C5CC04531B.

(11) Toohey, K. S.; Sottos, N. R.; Lewis, J. A.; Moore, J. S.; White, S. R. Self-Healing Materials with Microvascular Networks. Nat. Mater. 2007, 6 (8), 581-585. https://doi.org/10.1038/nmat1934.

(12) Oehlenschlaeger, K. K.; Mueller, J. O.; Brandt, J.; Hilf, S.; Lederer, A.; Wilhelm, M.; Graf, R.; Coote, M. L.; Schmidt, F. G.; Barner-Kowollik, C. Adaptable Hetero Diels-Alder Networks for Fast Self-Healing under Mild Conditions. Adv. Mater. 2014, 26 (21), 3561-3566. https://doi.org/10.1002/adma.201306258.

(13) Chakma, P.; Konkolewicz, D. Dynamic Covalent Bonds in Polymeric Materials. Angew. Chem. Int. Ed. 2019, 58, 9682-9695. https://doi.org/10.1002/anie.201813525.

(14) Herbst, F.; Döhler, D.; Michael, P.; Binder, W. H. Self-Healing Polymers via Supramolecular Forces. Macromol. Rapid Commun. 2013, 34 (3), 203-220. https://doi.org/10.1002/marc.201200675.

(15) Enke, M.; Doehler, D.; Bode, S.; Binder, W. H.; Hager, M. D.; Schubert, U. S. Intrinsic 
Self-Healing Polymers Based on Supramolecular Interactions: State of the Art and Future Directions. Adv. Polym. Sci. 2016, 273, 59-112. https://doi.org/10.1007/12_2015_345.

(16) Krogsgaard, M.; Nue, V.; Birkedal, H. Mussel-Inspired Materials: Self-Healing through Coordination Chemistry. Chem. Eur. J. 2016, 22, 844-857. https://doi.org/10.1002/chem.201503380.

(17) Yanagisawa, Y.; Nan, Y.; Okuro, K.; Aida, T. Mechanically Robust, Readily Repairable Polymers via Tailored Noncovalent Cross-Linking. Science 2018, 359 (6371), 72-76. https://doi.org/10.1126/science.aam7588.

(18) Hentschel, J.; Kushner, A. M.; Ziller, J.; Guan, Z. Self-Healing Supramolecular Block Copolymers. Angew. Chem. Int. Ed. 2012, 51 (42), 10561-10565. https://doi.org/10.1002/anie.201204840.

(19) Williams, G. A.; Ishige, R.; Cromwell, O. R.; Chung, J.; Takahara, A.; Guan, Z. Mechanically Robust and Self-Healable Superlattice Nanocomposites by Self-Assembly of Single-Component "Sticky" Polymer-Grafted Nanoparticles. Adv. Mater. 2015, 27 (26), 39343941. https://doi.org/10.1002/adma.201500927.

(20) Song, Y.; Liu, Y.; Qi, T.; Li, G. L. Towards Dynamic but Supertough Healable Polymers through Biomimetic Hierarchical Hydrogen-Bonding Interactions. Angew. Chem. Int. Ed. 2018, 57 (42), 13838-13842. https://doi.org/10.1002/anie.201807622.

(21) Wang, W.; Lu, W.; Goodwin, A.; Wang, H.; Yin, P.; Kang, N.-G.; Hong, K.; Mays, J. W. Recent Advances in Thermoplastic Elastomers from Living Polymerizations: Macromolecular Architectures and Supramolecular Chemistry. Prog. Polym. Sci. 2019, 95, 1-31. https://doi.org/10.1016/j.progpolymsci.2019.04.002.

(22) Cui, J.; Daniel, D.; Grinthal, A.; Lin, K.; Aizenberg, J. Dynamic Polymer Systems with Self-Regulated Secretion for the Control of Surface Properties and Material Healing. Nature Mater. 2015, 14 (8), 790-795. https://doi.org/10.1038/nmat4325.

(23) Varley, R. J.; Shen, S.; van der Zwaag, S. The Effect of Cluster Plasticisation on the Self Healing Behaviour of Ionomers. Polymer 2010, $51 \quad$ (3), 679-686. https://doi.org/10.1016/j.polymer.2009.12.025.

(24) Falco, G.; Simonin, L.; Pensec, S.; Dalmas, F.; Chenal, J.-M.; Bouteiller, L.; Chazeau, L. Linear and Nonlinear Viscoelastic Properties of Segmented Silicone-Urea Copolymers: Influence of the Hard Segment Structure. Polymer 2020, 186, 122041. https://doi.org/10.1016/j.polymer.2019.122041.

(25) Isare, B.; Pensec, S.; Raynal, M.; Bouteiller, L. Bisurea-Based Supramolecular Polymers: From Structure to Properties. C. R. Chim. 2016, 19, 148-156. https://doi.org/10.1016/j.crci.2015.06.013.

(26) Lortie, F.; Boileau, S. B.; Bouteiller, L.; Chassenieux, C.; Laupretre, F. Chain StopperAssisted Characterization of Supramolecular Polymers. Macromolecules 2005, 38 (12), 52835287. https://doi.org/10.1021/ma050168a. 
(27) Pinault, T.; Andrioletti, B.; Bouteiller, L. Chain Stopper Engineering for Hydrogen Bonded Supramolecular Polymers. Beilstein J. Org. Chem. 2010, 6, 869-875. https://doi.org/10.3762/bjoc.6.102.

(28) Molar masses measured by SEC are reported in Table S1.

(29) Our objective was to probe autonomous self-healing (i.e. at room temperature), but of course healing is accelerated at higher temperatures as shown by the temperature evolution of the terminal relaxation time (see below).

(30) The case of the blend $\mathbf{T} 3$ will be discussed below.

(31) Yilgör, E.; Yilgör, İ.; Yurtsever, E. Hydrogen Bonding and Polyurethane Morphology. I. Quantum Mechanical Calculations of Hydrogen Bond Energies and Vibrational Spectroscopy of Model Compounds. Polymer 2002, 43 (24), 6551-6559. https://doi.org/10.1016/S00323861(02)00567-0.

(32) Rubinstein, M.; Colby, R. H. Polymer Physics; Oxford University Press: Oxford; New York, 2003.

(33) Note that this value is derived from molar mass measurement by size exclusion chromatography and is therefore not an absolute value.

(34) Callies, X.; Vechambre, C.; Fonteneau, C.; Herbst, F.; Chenal, J.-M.; Pensec, S.; Chazeau, L.; Binder, W. H.; Bouteiller, L.; Creton, C. Effects of Multifunctional Crosslinkers on Rheology and Adhesion of Soft Nanostructured Materials. Soft Matter 2017, 13, 7979-7990. https://doi.org/10.1039/C7SM01304C.

(35) Relaxation times for the IPDI based copolymers are higher than TDI based copolymers, so that the difference between matrix and blend are visible in stress relaxation curves.

(36) Zhang, A.; Yang, L.; Lin, Y.; Yan, L.; Lu, H.; Wang, L. Self-Healing Supramolecular Elastomers Based on the Multi-Hydrogen Bonding of Low-Molecular Polydimethylsiloxanes: Synthesis and Characterization. J. Appl. Polym. Sci. 2013, 129 (5), 2435-2442. https://doi.org/10.1002/app.38832.

(37) Tazawa, S.; Shimojima, A.; Maeda, T.; Hotta, A. Thermoplastic Polydimethylsiloxane with L-phenylalanine-based Hydrogen-bond Networks. J. Appl. Polym. Sci. 2018, 135 (24), 45419. https://doi.org/10.1002/app.45419.

(38) Yilgör, E.; Yilgör, I. Silicone Containing Copolymers: Synthesis, Properties and Applications. Prog. Polym. Sci. 2014, 39 (6), 1165-1195. https://doi.org/10.1016/j.progpolymsci.2013.11.003. 